

M. J. Oddy,

M. J. Jones,

C. J. Pendegrass,

J. R. Pilling,

J. A. Wimhurst

From the Norfolk

and Norwich

University Hospital

NHS Trust, Norwich,

England

\title{
Assessment of reproducibility and accuracy in templating hybrid total hip arthroplasty using digital radiographs
}

In 20 patients undergoing hybrid total hip arthroplasty, the reproducibility and accuracy of templating using digital radiographs were assessed. Digital images were manipulated using either a ten-pence coin as a marker to scale for magnification, or two digital-line methods using computer software. On-screen images were templated with standard acetate templates and compared with templating performed on hard-copy digital prints.

The digital-line methods were the least reliable and accuracy of sizing compared with the inserted prostheses varied between $-1.6 \%$ and $+10.2 \%$. The hard-copy radiographs showed better reproducibility than the ten-pence coin method, but were less accurate with $3.7 \%$ undersizing. The ten-pence coin method was the most accurate, with no significant differences for offset or acetabulum, and undersizing of only $0.9 \%$.

On-screen templating of digital radiographs with standard acetate templates is accurate and reproducible if a radiopaque marker such as a ten-pence coin is included when the original radiograph is taken.

Pre-operative planning for total hip arthroplasty may require templating of radiographs. The aims are to assess the optimal size for acetabular press-fit, select appropriately-sized femoral components, improve leg-length equalisation and offset, optimise the position of the implant and avoid complications such as fracture. ${ }^{1-4}$ Templating requires consistency of the methods by which radiographs are taken and produced and knowledge of the magnification of the images and templates. It has been shown that radiopaque markers of known size used to determine image magnification can significantly improve the accuracy of templating. ${ }^{5}$

The introduction of digital technology in our hospital, with on-screen only radiographs, has complicated the pre-operative templating process, since the user has the ability to manipulate the images and thus alter the magnification. In an attempt to produce more useful radiographs, the digital images have been printed as hard copies after enlargement to give a best-fit image to simulate a traditional radiograph. Although these hard copies may aid templating they defeat the point of digital technology and are not produced according to a consistent protocol to standardise the size of the image.

We have performed a study to compare hard copies of digital images with three on-screen computer methods of manipulation of magni- fication of the image, using templating for hybrid total hip arthroplasty as the subject of our investigation. We have examined the different methods for reproducibility and also for accuracy, compared with the prostheses implanted subsequently. We tested the hypothesis that pre-operative templating with standard acetate templates using on-screen digital radiographs was more accurate and reproducible when a scale marker of known size was included to assess magnification, compared with using digital hard copies or other onscreen images in which no external scale marker was included.

\section{Patients and Methods}

Local ethical approval was obtained for a study on 20 patients undergoing primary total hip arthroplasty. A protocol was agreed by radiologists, radiographers, clinic nursing staff and the orthopaedic surgeon in order that the pre-operative radiographs could be performed with a ten-pence coin taped to the lateral aspect of the hip at the level of the most prominent part of the greater trochanter by the Senior House Officer ( $\mathrm{SHO}$ ) or nurse at the pre-assessment clinic (Fig. 1). The radiographs were taken as a consecutive series, although the subsequent operations were not performed in series. The decision to perform a hybrid arthroplasty was based on the assessment by 


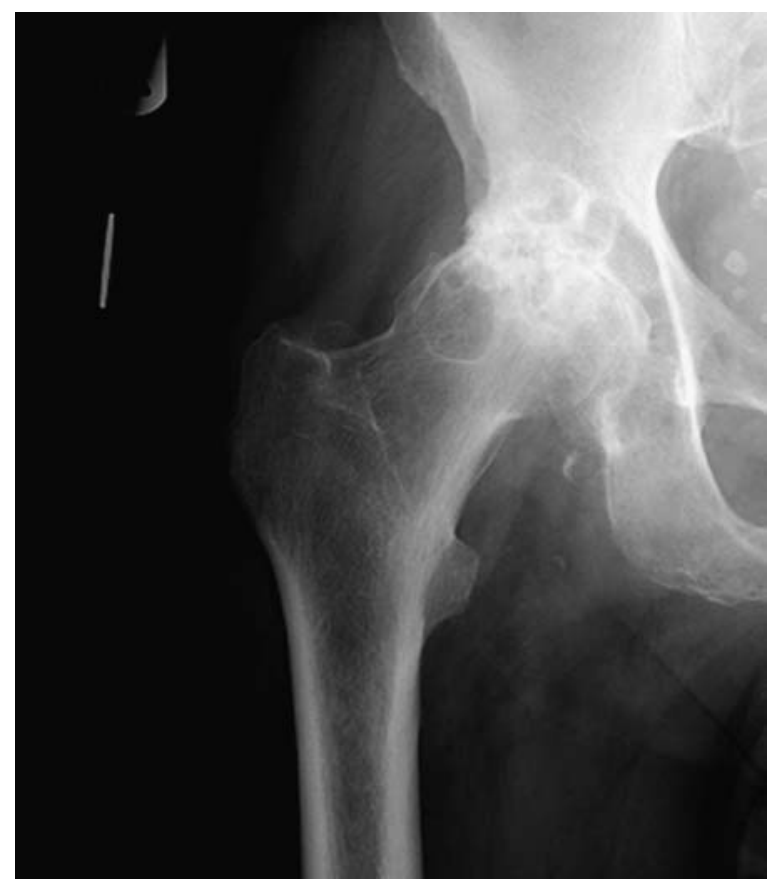

Fig. 1

Pre-operative anteroposterior radiograph showing the ten-pence coin marker on the skin.

the consultant surgeon at the pre-operative clinic. A standard pre-operative series of radiographs consisted of an anteroposterior (AP) view of the pelvis, and an AP and lateral view of the hip. Digital radiographs were taken according to a standard protocol with the patellae facing vertically if allowed by the internal rotation of the hip. The images were printed out as best-fit hard-copy radiographs in addition to being produced on-screen using the radiology server in our hospital (GE PACS Web Server-Image \& Information Gateway; Path Speed Web 8.1; GE Medical Systems, Wallingford, Connecticut).

All the pre-operative hard-copy radiographs were templated by three grades of surgeon (consultant, JAW, specialist registrar, MJO and Senior house officer, MJJ) who were blinded to the identity of the patient in order to assess interobserver variability. One investigator $(\mathrm{MJJ})$ repeated the templating process at an interval of two weeks to assess intra-observer variability. The on-screen images were templated using three different methods to correct for magnification.

General templating method. The AP radiographs of the hip were templated pre-operatively using a single set of acetate templates for a hybrid total hip arthroplasty on both the digital computer-screen images and the hard-copy radiographs. The templates were implant-specific and included a magnification factor of $20 \%$ as supplied by the manufacturers (Reflection; Smith \& Nephew, Cambridge, United Kingdom, and Exeter V40; Stryker Howmedica Osteonics,

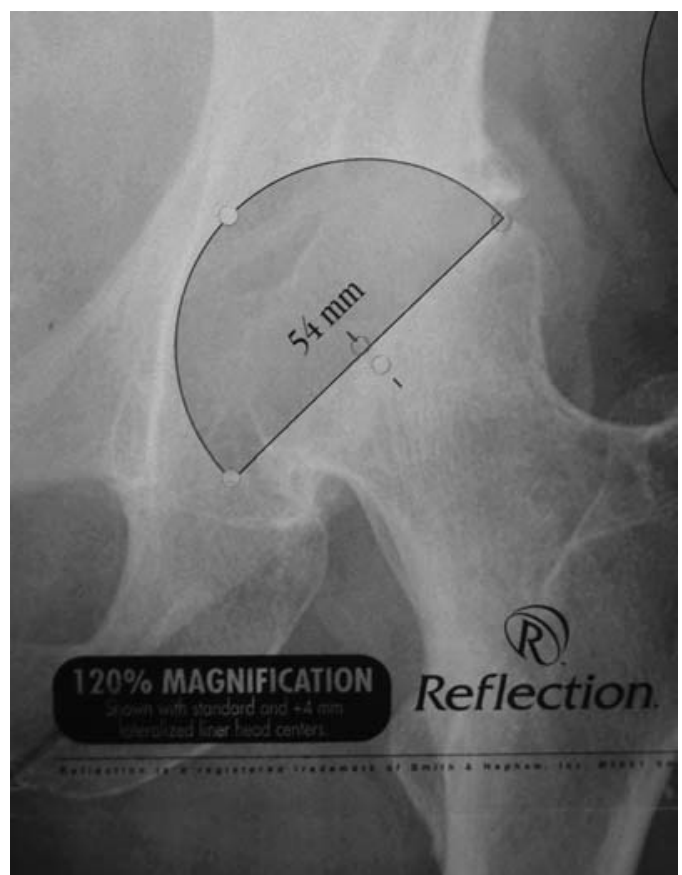

Fig. 2

Computer image of a hip with the acetabular template applied to the computer screen.



Fig. 3

Computer image with the acetate stem template applied to the computer screen. 
Table I. ICC* values for intra-observer variability

\begin{tabular}{lllll}
\hline & & \multicolumn{2}{l}{ Digital line } & \\
\cline { 3 - 4 } & Ten-pence & Method 1 & Method 2 & Hard-copy \\
\hline Acetabulum & 0.90 & 0.95 & 0.80 & 0.85 \\
Femoral offset & 0.88 & 0.95 & 0.90 & 0.88 \\
Template offset & 0.80 & 1.00 & 0.77 & 1.00 \\
Size of femoral component & 0.70 & 0.89 & 0.90 & 0.91 \\
\hline * & & &
\end{tabular}

* ICC, intraclass correlation coefficient

Table II. ICC* values for inter-observer variability

\begin{tabular}{lllll}
\hline & & \multicolumn{2}{l}{ Digital line } \\
\cline { 3 - 4 } & Ten-pence & Method 1 & Method 2 & Hard-copy \\
\hline Acetabulum & 0.68 & 0.43 & 0.47 & 0.65 \\
Femoral offset & 0.76 & 0.78 & 0.64 & 0.85 \\
Template offset & 0.67 & 0.70 & 0.63 & 0.85 \\
Size of femoral component & 0.72 & 0.34 & 0.62 & 0.83 \\
\hline
\end{tabular}

* ICC, intraclass correlation coefficient

Table III. ICC ${ }^{*}$ values for variability of derived difference between templated results and inserted prostheses

\begin{tabular}{lllll}
\hline & & \multicolumn{2}{l}{ Digital line } & \\
\cline { 3 - 4 } & Ten-pence & Method 1 & Method 2 & Hard-copy \\
\hline Acetabulum & 0.79 & 0.54 & 0.48 & 0.69 \\
Template offset & 0.68 & 0.73 & 0.46 & 0.85 \\
Size of femoral component & 0.81 & 0.51 & 0.49 & 0.92 \\
\hline
\end{tabular}

* ICC, intraclass correlation coefficient

Newbury, United Kingdom). The acetabular component was sized so that the inferior aspect was level with the base of the teardrop and the medial aspect approximated the ilio-ischial line, aiming for maximum cover with an inclination angle of approximately $45^{\circ}$ (Fig. 2).

On the femoral side, three templating measurements were examined. The femoral offset was a scalar perpendicular distance measurement taken from a line projected cephalad from the centre of the femoral shaft to the centre of rotation of the femoral head. The template offset was a best-fit obtained using the template profile for the three available offsets $(37.5 \mathrm{~mm}, 44 \mathrm{~mm}$ and $50 \mathrm{~mm}$ ) in order to reproduce the patient's anatomy, while the size of the femoral component was assessed as the template profile which best filled the femoral metaphysis while allowing for a cement mantle of $2 \mathrm{~mm}$, corresponding to the first marked graduation on the template and the recommended minimum cement mantle ${ }^{6}$ (Fig. 3).

The ten-pence coin method. The size of the image was enlarged using the computer software until the size of the ten-pence coin measured its true size of $24.5 \mathrm{~mm}$, using the magnified ruled line scale on the acetate templates.

Digital line, method 1. A line $50 \mathrm{~mm}$ long was drawn using the computer software on the screen and the image size was adjusted so that the digital line equalled a distance of 50 $\mathrm{mm}$ on the acetate template scale. Once the digital-line scale method had been used to adjust the size of the image and templating had been performed, the size of the tenpence coin on the image was measured using the template linear scale and the value recorded.

Digital line, method 2. A similar $50 \mathrm{~mm}$ line was drawn using the computer software, and the image was adjusted so that the digital line equalled a distance of $50 \mathrm{~mm}$ on a normal ruler. Using this digital-line method, the size of the ten-pence coin was measured using both a normal ruler, and also an acetate template scale. The actual implanted component sizes for the acetabulum, offset and size of the femoral component were retrieved from theatre records for the analysis.

Statistical analysis. The data were analysed using SPSS for Windows version 12.0 (SPSS Inc., Chicago, Illinois). Intraand inter-observer effects were analysed using intraclass correlation coefficients (ICC) to examine the reproducibility for an observer between methods and between different observers for each method. An ICC analysis was also performed to compare the data for the actual prostheses inserted with the templated values. The analysis created a model incorporating all of the factors which affected the variance so that higher ICC values reflected variation not associated with either the observer or method. Values closer to 1.00 were, therefore, a reflection of improved reproducibility. In addition to ICC analyses, assessment of the differ- 
ences between methods for the templated results as performed using the paired $t$-test and the results were considered to be significant at $\mathrm{p}<0.05$.

\section{Results}

The ICC values for the initial and repeat measurements reflecting the intra-observer variation are shown in Table I. All methods showed good reproducibility with ICC values $>0.70$ for all parameters. ICC values for the inter-observer variation between the three grades of observer are shown in Table II. The hard-copy radiographs and the ten-pence coin method both showed acceptable to good reproducibility for all four parameters with ICC values of approximately 0.70 or more, while the two digital-line methods were less reliable with poor ICC values of $<0.50$ for the acetabulum and of the femoral component size. Using a paired $t$-test to compare the templated results, for all four parameters the values of the digital-line method 1 were significantly higher than those of the other three methods (all $p<0.05$ ). The results for the ten-pence coin method and the digital-line method 2 were very similar for most parameters, being significantly different only for the acetabulum when the digital-line method 2 was significantly lower $(\mathrm{p}<0.046)$.

ICC values comparing the derived differences between the templated results with the actual prostheses inserted are given in Table III. Both digital-line methods showed poor reproducibility with only one parameter of template offset having a value $>0.70$ for digital-line method 1 and none for method 2. Both the ten-pence coin and hard-copy methods showed good correlation with the inserted prostheses for all four parameters. The templated results for the ten-pence coin method and the digital line method 2 were not significantly different for either the acetabulum or offset $(p>$ 0.05 ) compared with the inserted prostheses, but were for the size of the femoral component $(\mathrm{p}<0.05)$. The templated values using the hard-copy radiographs were not significantly different from the inserted prostheses for either the offset or size of the femoral component (both $p>0.05$ ), but were for the acetabulum $(\mathrm{p}<0.008)$. Using the digitalline method 1, all the parameters templated were significantly different from and therefore correlated poorly with the actual prostheses inserted (all $\mathrm{p}<0.05$ ).

When the digital image was adjusted according to the digital-line method 1, measurement using an acetate template scale of the size of the ten-pence coin as seen in the onscreen image produced a mean oversized magnification of $14.5 \%(95 \%$ confidence interval (CI) +13.0 to +16.0$)$ compared with the true size of a ten-pence coin. For the digitalline method 2, when the size of the ten-pence coin was measured using an acetate template ruler, the image was undersized by a mean of $0.5 \%(95 \% \mathrm{CI}-1.3$ to +3.0$)$. For the digital-line method 2, when the ten-pence coin was measured with a normal ruler there was a mean oversized magnification of $16.7 \%$ (95\% CI +15.7 to +17.7).

A template-implant magnification ratio expressed as a percentage was derived, based on the mean size of the tem- plated acetabular prostheses compared with that of the actual inserted acetabular prostheses. The relative size alterations per method were $10.2 \%(95 \% \mathrm{CI}+6.4$ to +14.0$)$ oversize for the digital-line method 1, 1.6\% (95\% CI -4.6 to 1.4 ) undersize for the digital-line method 2, $0.9 \%(95 \%$ CI -3.5 to +3.3 ) undersize for the ten-pence method and $3.7 \%(95 \%$ CI-1.1 to -6.4$)$ undersize for the hard-copy method.

\section{Discussion}

Accuracy in templating using standard radiographs is multifactorial and can be as low as $50 \%$ for femoral components ${ }^{7}$ and $65 \%$ for uncemented acetabular components. ${ }^{4}$ Conventional radiographs taken with a source-to-plate distance of $1 \mathrm{~m}$ lead to apparent image magnification of approximately $20 \%, 5,8$ which results in the standard practice of implant-specific acetate templates with pre-set magnifications. The magnification of a standard radiograph is affected by the source-to-object distance, and also the object-to-plate distance. ${ }^{1,5}$ Attempts to control magnification are generally impractical for radiographers and have been shown not to be reproducible, even on the same patient, with a strong tendency for over-magnification. ${ }^{9}$ The appearance of the image can also be affected by rotation of the femur, although the effect becomes less significant distally in the femur. ${ }^{10}$ Conn et $\mathrm{al}^{5}$ showed that the use of a ten-pence piece as a radiopaque marker to assist with magnification improved the templating accuracy using standard radiographs from $59.5 \%$ to $68.8 \%$.

Digital radiograph technology is increasingly being introduced into hospitals. One of its main advantages in association with a Picture Archiving and Communication System is the ability to adjust the processed image to manipulate quality. ${ }^{11}$ Specifically, the film reject rate has been shown to be reduced from $2.1 \%$ with conventional radiography, to $1.3 \%$ with digital technology which has further implications in reducing patient radiation exposure. ${ }^{12}$ On-screen templating for total joint arthroplasty requires specific digital software which is an extra cost and not currently available in our institution. A recent review presenting a digital templating algorithm still describes as being integral to the process the determination of magnification with a marker of known dimension at the time the radiograph is made. ${ }^{13}$ White and Shardlow ${ }^{3}$ compared conventional and digital hard-copy radiographs and showed that templated values for the size of the femoral component correlated only moderately in agreement, but correlated well for offset. They highlighted the fact that with digital radiograph prints using newer technology the effect of magnification needs to be considered with respect to templating for arthroplasty. ${ }^{3}$ No study to date has examined on-screen digital templating with standard acetate templates and compared this with hard-copy digital prints as we have done in our study. The true implications on accuracy in templating with conventional versus digital radiology have yet to be shown. 
Our study has examined four different methods of manipulating image magnification using digital radiograph technology in an attempt to find the most reproducible and accurate method to aid templating for hybrid total hip arthroplasty, without using specific digital templating software. We used standard acetate templates with a magnification factor of $20 \%$ as standard. These templates included a magnified ruled line scale. All four methods showed good intra-observer correlation using the observer least experienced in pre-operative templating ( $\mathrm{SHO}$ ).

The least accurate and reproducible was the on-screen digital-line method 1 , while the ten-pence coin method using a marker of known size alone proved to be the most accurate with undersizing of only $0.9 \%$.

Manipulating the digital line against a normal ruler in the digital-line method 2 produced a similar poor degree of inter-observer variability as in method 1 . However, the templated results very closely matched the results for the tenpence coin method. The differences in results using the two digital-line methods highlight the significance of magnification within the digital technology system, which has significant implications when templating press-fit acetabular components in which the usual oversizing is only 1 to $2 \mathrm{~mm}$ above the final reamer, and oversizing beyond that significantly increases the risk of fracture. In fact, the digital software in our department has been configured such that the digital-line software is applied as a non-magnified 'real scale' to an image which has already been magnified by about $20 \%$ through the process of its acquisition. Therefore the digital-line method 1 subsequently uses a magnified scale to enlarge an image which has already been magnified. Method 2 assumes that the image has already been magnified and thus the drawn digital line is configured against a normal ruler. Hence, when the ten-pence coin in the method 2 image is measured with a normal ruler, it appears to be magnified (mean 16.7\%), but when measured with an already magnified template scale it appears to be accurate (mean undersize of $0.5 \%$ ).

The hard-copy radiographs were prints of the digital image scaled to best-fit standard radiograph films in order to simulate a traditional radiograph. Templating reproducibility was marginally better than for the ten-pence method which may reflect the overall user familiarity with a radiographic film rather than a computer screen. However, the overall accuracy compared with the implanted prostheses was not as good when compared with the ten-pence coin method. The hard-copy method resulted in the templated results being undersized by a mean of $3.7 \%$ compared with that of the actual prostheses. This is of a similar proportion to findings in studies comparing standard and digitallyacquired radiographs in which the digital radiographic prints resulted in an undersizing magnification of $97 \% .^{3}$

Accurate and reproducible direct on-screen templating of digital radiographs can be performed with existing acetate templates, provided that either the radiographs are taken with a radiopaque marker of known size placed in a standard position, or the method by which the computer software is configured to the image is known. However, manipulating image size against a marker of known size is more reproducible than using a digital-line technique. We feel that while on-screen radiographs have superseded hard copies at our institution, the current results support the inclusion of a known size marker, such as a ten-pence coin, at the time of acquisition of the digital radiographs, to allow accurate templating with current acetate templates. A follow-up study comparing the ten-pence coin method with acetate templates against dedicated digital templating software is planned.

We are grateful to Richard Morris, Department of Primary Care \& Population Sciences, The Royal Free and University College Medical School for advice with statistical analysis.

No benefits in any form have been received or will be received from a commercial party related directly or indirectly to the subject of this article.

\section{References}

1. Gorski JM, Schwartz L. A device to measure x-ray magnification in preoperative planning for cementless arthroplasty. Clin Orthop 1986;202:302-6.

2. Knight JL, Atwater RD. Preoperative planning for total hip arthroplasty. J Arthroplasty 1992;7(Suppl):403-9.

3. White SP, Shardlow DL. Effect of introduction of digital radiographic techniques on pre-operative templating in orthopaedic practice. Ann R Coll Surg Eng/2005;87:53-4.

4. Gonzalez Della Valle A, Slullitel G, Piccaluga F, Salvati EA. The precision and usefulness of preoperative planning for cemented and hybrid primary total hip arthroplasty. J Arthroplasty 2005;20:51-8.

5. Conn KS, Clarke MT, Hallett JP. A simple guide to determine the magnification of radiographs and to improve the accuracy of preoperative templating. J Bone Joint Surg [Br] 2002;84-B:269-72.

6. Exeter Total Hip System. Manual of operative technique V40 ${ }^{T M}$. Stryker Howmedica Osteonics, Stryker Europe, Newbury, Berks, RG14 5EG, UK.

7. Carter LW, Stovall DO, Young TR. Determination of accuracy of preoperative templating of noncemented femoral prostheses. J Arthroplasty 1995;10:507-13.

8. Clarke IC, Gruen T, Matos M, Amstutz HC. Improved methods for quantitative radiographic evaluation with particular reference to total-hip arthroplasty. Clin Orthop 1976;121:83-91.

9. Linelau L, Dokter G, Peene P. Radiological aspects in preoperative planning and postoperative assessment of cementless total hip arthroplasty. Acta Orthop Belgica 1993:59:163-7.

10. Eckrich SGJ, Noble PC, Tullos HS. Effects of rotation on the radiographic appearance of the femoral canal. J Arthroplasty 1994;9:419-26.

11. Strickland NH. PACS (picture archiving and communication systems): filmless radiology. Arch Dis Child 2000;83:82-6.

12. Lau S-L, Mak AS-H, Lam W-T, Chau C-K, Lau K-Y. Reject analysis: a comparison of conventional film-screen radiography and computed radiography with PACS. Radiography 2004;10:183-7.

13. Bono JV. Digital templating in total hip arthroplasty. J Bone Joint Surg [Am] 2004; 86-A(Suppl 2):118-22. 